ANALYSE

Die Lage der politischen Opposition in Polen

Janusz A. Majcherek, Akademia Sztuk Teatralnych, Krakau

- UMFRAGEN

Parteipräferenzen und Politikerratings

CHRONIK

15. - 28. Februar 2022

Forschungsstelle Osteuropa an der Universität Bremen 


\section{Die Lage der politischen Opposition in Polen}

Janusz A. Majcherek, Akademia Sztuk Teatralnych, Krakau

DOI: $10.31205 /$ PA.289.01

\section{Zusammenfassung}

Der fortschreitende Niedergang des demokratischen Rechtsstaates, vorangetrieben von den in Polen regierenden Rechtspopulisten, wird seit etlichen Monaten von Zersetzungsprozessen im Regierungslager sowie sinkender gesellschaftlicher Unterstützung für dessen Handeln begleitet. Dies lenkt das Interesse der öffentlichen Meinung zunehmend auf die politische Alternative, d. h. die aktuelle Opposition. Diese bleibt, trotz zunehmender und lauter werdender Aufforderungen der Wähler und Kommentatoren zu mehr Einigkeit, zersplittert und gespalten.

$\mathrm{Z}$ unächst ist festzuhalten, dass sich die regierenden Rechtspopulisten fast alle öffentlichen Institutionen untergeordnet haben und sie für eine koordinierte Anti-Oppositions-Propaganda einsetzen. Insbesondere das öffentliche Polnische Fernsehen (TVP) zeichnet sich durch systematische Negativkampagnen aus, die gegen die Hauptpersonen und -gruppierungen der Opposition gerichtet sind. Leider hat auch der deutsche Medienkonzern Verlagsgruppe Passau dazu beigetragen, diese Vorgehensweise zu erleichtern, denn er verkaufte etliche regionale Tageszeitungen, Wochenzeitungen und online-Portale an den polnischen Mineralölkonzern Orlen, der von Akteuren, die der Regierungspartei nahe stehen, kontrolliert wird (siehe Polen-Analysen Nr. 269, https://www.laender-analysen.de/polen-analysen/269/). Die aufgekauften Zeitungen wurden der Propagandamaschine des Regierungslagers angeschlossen.

Von Hackern enthüllte E-Mails privater E-Mailkonten von Mitgliedern der Regierungsmannschaft zeigten, dass es ein permanenter und wesentlicher Bestandteil der Beratungen und Besprechungen des Regierungslagers ist, sich selbst ein positives Image zu geben sowie Möglichkeiten zu ersinnen, die Opposition anzuschwärzen. Sowohl im Hintergrund als auch in der Regierung arbeitet eine spezialisierte Gruppe von Experten für politisches Marketing und Wettbewerb, die sich fortlaufend Propagandakampagnen ausdenkt. Dabei kommen auch antideutsche und antieuropäische Narrationen zum Einsatz, welche die Politiker der Opposition als Diener deutscher und europäischer, aber nicht polnischer Interessen diskreditieren sollen.

Das Hauptziel dieser Propagandaattacken ist Donald Tusk. Der ehemalige Ministerpräsident Polens stammt aus einer Familie, die in der ehemaligen Freien Stadt Danzig (heute Gdańsk) lebte; von 2014 bis 2019 übte er das Amt des EU-Ratspräsidenten aus. Er eignet sich also dafür, die deutschen und europäischen Verflechtungen zu repräsentieren sowie den angeblichen Einfluss der Opposition auf Berlin und Brüssel, wobei er als ihr wichtigster Akteur angesehen wird.

\section{Das Kräfteverhältnis im Oppositionslager} Tusk steht gegenwärtig an der Spitze der stärksten Oppositionspartei, der Bürgerplattform (Platforma Obywatelska-PO). Er hat diesen Platz vor einigen Monaten erneut eingenommen, als er seine Mission in der Europäischen Union beendet hat und in die polnische Politik zurückgekehrt ist. Zuvor hatte er seine Partei im Zustand der Krise zurückgelassen, die von mehreren Wahlniederlagen sowie der scharfen Konkurrenz vonseiten der oppositionellen Gegner hervorgerufen worden war. Seine Rückkehr aus Brüssel belebte die $P O$ wieder und stärkte ihre Spitzenposition im Oppositionslager mit Umfragewerten, die sich um 25 Prozent bewegen. So scheint sich seine persönliche Position - er ist wahrscheinlich der erfahrenste und in der internationalen Politik am stärksten verwurzelte polnische Politiker günstig auf die von ihm geführte Partei auszuwirken. Die $P O$ ist liberal-konservativ; ihre Europaabgeordneten gehören zur Fraktion der Europäischen Volkspartei (EVP), der auch die CDU angehört. Tusk steht zurzeit dieser Fraktion vor.

Platz zwei auf der Unterstützungsskala der AntiRegierungswähler belegt eine erst im Jahr 2020 vom damaligen Präsidentschaftskandidaten Szymon Hołownia initiierte Gruppierung. Hołownia ist relativ jung (45 Jahre) und ein Anfänger in der Politik, gleichzeitig aber ein bekannter katholischer Publizist und TV-Moderator, der 2020 recht unerwartet seine Anwartschaft auf das Präsidentenamt verkündete und im landesweit durchgeführten ersten Wahlgang knapp 14 Prozent erzielte. Er kam nicht in den entscheidenden zweiten Wahlgang (in dem der amtierende Präsident und Kandidat des rechtskonservativen Lagers, Andrzej Duda, nur knapp den für die $P O$ angetretenen Stadtpräsidenten von Warschau, Rafał Trzaskowski, schlug), aber es gelang ihm mit der Unterstützung seiner Wähler, eine politische Bewegung ins Leben zu rufen, deren Umfragewerte rasch stiegen. Zeitweilig übertrafen sie die der $P O$ und Hołownias Gruppierung besetzte die 
Führungsposition im oppositionellen Lager. Seit Tusks Rückkehr auf die polnische politische Bühne und der Stärkung der $P O$ liegt die Unterstützung für Hołownias Bewegung Polen 2050 (Polska 2050) zwischen 10 und 15 Prozent. Da sie relativ neu ist, hat sie praktisch keine parlamentarische Repräsentanz, zumal ihr nur vereinzelt Personen aus anderen Parteien mit ihren Abgeordnetenmandaten beitraten, und ist in staatlichen Institutionen, deren Entscheidungsgremien gewählt werden, noch nicht vertreten.

Die Übernahme von Politikern und Akteuren anderer, länger bestehender oppositioneller Gruppierungen ist einer der Vorwürfe, den deren Anführer Hołownia machen. Er selbst versichert, dass er im Stande sei, Akteure und Wähler der Regierungspartei Recht und Gerechtigkeit (Prawo i Sprawiedliwość - PiS) für Polen $2050 \mathrm{zu}$ gewinnen und so das summarische und proportionale Ergebnis der Opposition gegenüber dem der $P i S$ zu verbessern. Das erwies sich allerdings als unhaltbare Erklärung, denn Untersuchungen zeigen, dass ein verschwindend geringer Anteil der PiS-Anhänger Polen 2050 als zweite Option betrachtet.

Hołownia selbst hatte noch vor einigen Jahren recht konservative, wenngleich tolerante Ansichten im Geiste eines offenen Katholizismus und trat für diese auch in seiner Publizistik ein. In letzter Zeit mildert er sie $a b$, zum Beispiel in Fragen der Rechte für LGBT+-Personen oder der Zulässigkeit von Abtreibung, Themen, die Gegenstand heftiger weltanschaulicher Kontroversen sind. In der aktuell für Polen grundlegenden politischen Frage der Notwendigkeit, sich gegen den fortschreitenden Autokratismus zu stellen und für die Verteidigung des demokratischen Rechtsstaates einzustehen, vertritt Hołownia prodemokratische und prorechtsstaatliche Ansichten. Dabei ist er bemüht, sie als eigenständig darzustellen, gesondert vom Rest der Opposition, von der er sich abgrenzt.

Dies liegt im Hauptproblem Hołownias und seiner Gruppierung begründet, und zwar der ideellen und weltanschaulichen Ähnlichkeit zur PO und dem sich daraus ergebenden starken Bedürfnis, Andersartigkeit zu markieren. Zu seinen Gunsten wirkt der Effekt des Neuen, der jedoch allmählich verfliegt. Immer häufiger richten sich vonseiten der oppositionellen öffentlichen Meinung Ermunterungen oder sogar Überredungsversuche an ihn, sich mit den Kräften der $P O$ zu verbinden. Hołownia hat dies bisher recht entschieden abgelehnt. Vermutlich will er seine politische Gruppierung in Wahlen testen; die nächsten wären die Parlamentswahlen, die regulär in anderthalb Jahren stattfinden würden.

Tief in der Krise steckt der linke politische Flügel. Er tritt zurzeit unter dem Schild Neue Linke (Nowa Lewica) auf, hinter dem der Zusammenschluss der Demokratischen Linksallianz (Sojusz Lewicy Demokratycznej-
SLD) und der Partei Gemeinsam (Razem) mit der an Bedeutung verlierenden Partei Frühling (Wiosna) des bekanntesten homosexuellen Politikers in Polen und aktuell Europaabgeordneten, Robert Biedroń, steht. Die Neue Linke erhielt in den Parlamentswahlen 2019 über zwölf Prozent; in den Meinungsumfragen liegt sie aber seit langem unter zehn Prozent und bewegt sich in Richtung Fünf-Prozent-Hürde (2015 hatte sie nicht in den Sejm einziehen können, weil sie die für Wahlbündnisse erforderlichen acht Prozent nicht erreicht hatte). Einer der Gründe für ihre nun schwachen Unterstützungswerte sind Reibereien, die den Zusammenschluss von SLD und Frühling begleiteten, aber auch die zweideutige Haltung gegenüber der rechtspopulistischen Regierung, die eine Politik verschwenderischer Sozialtransfers betreibt, was einem Teil der linken Aktivisten gefällt.

Das spektakulärste Beispiel war die Unterstützung linker Abgeordneter für den Landesaufbauplan, das von der polnischen Regierung der Europäischen Union vorgelegte Programm für den Einsatz von EU-Finanzmitteln, welche die EU ihren Mitgliedsländern mit dem Ziel bereit stellt, die infolge der Corona-Pandemie geschwächten Wirtschaften der EU-Staaten zu unterstützen. Die Europäische Kommission verweigerte dem polnischen Landesaufbauplan die Zustimmung. Gründe dafür sind die Verletzung der EU-Rechtsstaatsprinzipien durch Polen, die fehlende Anerkennung Polens der in diesem Bereich gesprochenen Urteile des Europäischen Gerichtshofes sowie inoffiziell auch die berechtigte Sorge, dass die Mittel den regierenden Rechtspopulisten dazu dienen würden, Wählerstimmen zu »kaufen«. Die Unterstützung der Linken für die polnische Regierung, entgegen der Warnungen der EU, rief einen Konflikt in der Opposition und negative Reaktionen in der linken Wählerschaft hervor.

Vor einigen Wochen verließen die linken Senatoren die Fraktion der Linken im Senat und schlossen sich neu zusammen.

Krisengebeutelt ist auch die schwächste der Oppositionsparteien, die Polnische Bauernpartei (Polskie Stronnictwo Ludowe - PSL). Einst zwei Legislaturperioden lang Regierungspartner der $P O$, distanziert sie sich seit langem vom ehemaligen Koalitionspartner und balanciert in Umfragen um die Fünf-Prozent-Hürde herum. Die regierenden Rechtspopulisten eroberten auf dem Land und in der Provinz eine umfangreiche gesellschaftliche Basis und verdrängten die früher dort dominierende $P S L$.

Außerhalb des Regierungslagers gibt es noch eine weitere parlamentarische Gruppierung, die rechtsnationalistische, libertäre und antieuropäische Konföderation (Konfederacja), die weltanschaulich mit der deutschen Partei AfD vergleichbar ist. Ihren oppositionellen Status lebt sie zweigleisig aus, indem sie sich sowohl von 
den anderen Oppositionsparteien abgrenzt als auch aus ihren radikalen Positionen heraus teilweise die Regierung angreift (zum Beispiel beim Auftritt gegen CoronaImpfungen oder der Bekämpfung jeglicher CoronaSchutzmaßnahmen). Insgesamt wird sie als potentieller Koalitionspartner der PiS betrachtet, sollte diese dauerhaft ihre parlamentarische Mehrheit verlieren.

\section{Perspektiven gemeinsamen Handelns}

Die bei den Wahlen zum Sejm (der unteren Parlamentskammer) geltende proportionale Sitzzuteilung nach dem D'Hondt-Verfahren, das die stärkeren Gruppierungen bevorzugt, und die Fünf-Prozent-Hürde veranlassen viele Kommentatoren und Wähler, die Bildung eines oppositionellen Blocks in Erwägung zu ziehen, der das nationalistisch-populistische Lager besiegen und vor allem garantieren soll, dass keine Stimme der oppositionellen Wähler vergeudet wird. Die Gegner argumentieren wiederum, dass die bisher unternommenen Versuche in diese Richtung in einem Fiasko mündeten.

In der Tat unternahm der damalige $P O$-Vorsitzende Grzegorz Schetyna vor den Wahlen zum Europäischen Parlament (2019) einen solchen Vereinigungsversuch. Auf seine Initiative hin bildete sich die Europäische Koalition (Koalicja Europejska), die 38,5 Prozent der Stimmen erhielt und gegen die regierende PiS verlor, die mehr als 45 Prozent erlangte. Allerdings startete damals auch Frühling von Robert Biedroń und bekam mehr als sechs Prozent (er selbst erlangte ein Abgeordnetenmandat) und befand sich die PiS zu dieser Zeit auf dem Gipfel ihrer politischen Stärke.

Die Europäische Koalition brach auseinander und zu den Parlamentswahlen in Polen noch im selben Jahr gingen die oppositionellen Gruppierungen gesondert, wobei sich um die $P O$ ein kleineres Bündnis unter dem Namen Bürgerkoalition (Koalicja Obywatelska - KO) bildete, was die liberale Partei Die Moderne (Nowoczesna), die kleine linke Gruppierung Initiative Polen (Inicjatywa Polska) sowie die schwache Partei Die Grünen (Zieloni) einband. Die PiS gewann auch diese Wahlen mit fast 45 Prozent, in absoluten Zahlen allerdings wurden knapp eine Million Stimmen mehr für die zersplitterte Opposition (ohne Konföderation) als für die PiS abgegeben. Bis heute ist das eines der Hauptargumente der Befürworter einer Vereinigung der Opposition.

Für die gleichzeitig stattgefundenen Wahlen zum Senat, bei denen das Mehrheitswahlrecht gilt, bildeten die Oppositionsparteien einen gemeinsamen Block (in jedem Ein-Mandats-Kreis wurde nur ein einziger und gemeinsamer Kandidat aufgestellt) und bekamen knapp die Mehrheit der Sitze dieser zweiten Parlamentskammer, die zu einer wichtigen Barriere für die autoritären Bestrebungen der regierenden PiS geworden ist.
Es ist wenig wahrscheinlich, dass es zu einer engeren Zusammenarbeit und Koordination der Aktivitäten der Oppositionsparteien noch vor den Parlamentswahlen in Ungarn im April 2022 kommen wird. Die Opposition dort hat einen gemeinsamen Block gebildet und dessen Ergebnis wird für die polnischen Politiker und Publizisten ein wichtiges Argument werden. Eine Niederlage wird vermutlich endgültig die Chancen auf Vereinigung der Opposition in Polen zunichtemachen.

Einige ihrer Vertreter berufen sich auf das Beispiel Tschechien, wo im vergangenen Jahr zwei oppositionelle Blocks die Populisten schlugen. Ein Fürsprecher dieser Lösung ist insbesondere der Parteichef der PSL, Władysław Kosiniak-Kamysz, der nahelegt, dass ein links-liberales Bündnis um die $P O$ einerseits und seine Partei mit Polen 2050 als konservativer Block andererseits nicht nur die oppositionelle Wählerschaft mobilisieren, sondern auch diejenigen Wähler gewinnen kann, die bisher für die rechten Populisten gestimmt haben.

Donald Tusk verfolgt am entschlossensten von allen Parteiführern die Idee der Vereinigung der Opposition, ruft damit aber Widerspruch bei potentiellen Partnern hervor, die in dieser Strategie den Wunsch sehen, sie zu dominieren. Dabei schließt Tusk recht entschieden die Linke aus einem eventuellen gemeinsamen Block aus, da sie nicht ausreichend regierungskritisch sei. Die PSL will trotz der gemeinsamen Regierungserfahrung mit der $P O$ und der Zugehörigkeit zur selben Fraktion im Europäischen Parlament keine Zusammenarbeit mit der $P O$ und versucht, um die Stimmen auf dem Land zu kämpfen, wo die $P O$ als Partei der Mittelklasse und der großstädtischen Wählerschaft (alle größeren Städte in Polen werden von $P O$-Vertretern oder Politikern, die von der $P O$ unterstützt werden, regiert) nicht populär ist. Hołownia wiederum will seine neue Gruppierung selbständig in den Wahlen prüfen.

Einer der Hauptgründe für die Zurückhaltung der kleineren Oppositionsparteien gegenüber dem Vorschlag der Kooperation ist demnach die Sorge, von der $P O$ dominiert zu werden. Sie zählen darauf, dass sie mit der Zeit ihre Umfragewerte verbessern und in eventuellen Verhandlungen mit Tusk noch vor den Wahlen eine stärkere Position werden einnehmen können. Immer mehr weist darauf hin, dass sich diese Rechnung als Fehlkalkulation erweisen kann. Ein Teil der Wähler signalisiert, dass, falls es nicht zur Aufstellung gemeinsamer Wahllisten für die gesamte Opposition kommt, er die stärkste oppositionelle Formation, also $P O / K O$, unterstützen wird. Das würde bedeuten, dass die übrigen Parteien Unterstützung verlieren und nicht gewinnen werden.

Sollte allerdings eine der Oppositionsparteien unter die Fünf-Prozent-Hürde fallen und nicht in den neuen Sejm einziehen, würde das eine Niederlage für die gesamte Opposition bedeuten, denn über ihren Sieg 
oder Niederlage werden wahrscheinlich nur wenige Prozent entscheiden.

Sehr wahrscheinlich ist dagegen die Bildung eines gemeinsamen Blocks für die Senatswahl, ähnlich wie im Jahr 2019. Ein erneuter Erfolg würde hier jedoch nicht bedeuten, der PiS die Regierungsmacht abzunehmen, denn der Senat hat keinen Einfluss auf die Regierungsbildung. Er kann allein Gesetzgebungsinitiativen verlangsamen oder blockieren sowie auf die Besetzung mancher staatlicher Institutionen Einfluss nehmen.

\section{Die Übernahme der Regierungsverantwortung wird nicht leicht} In letzter Zeit geben die Umfragen insgesamt der Opposition, sogar wenn sie so zersplittert bleibt, den Vorrang vor der PiS. Den Rechtspopulisten die Regierungsverantwortung abzunehmen, wird allerdings nicht leicht werden, da diese alle ergreifbaren Mittel einsetzen, um dies zu verhindern.

Wie unlängst bekannt wurde, haben die von der PiS kontrollierten Geheimdienste mit Hilfe der in Israel gekauften Überwachungssoftware Pegasus mutmaßlich den Chef des Wahlkampfstabes der PO im Jahr 2019 ausgespäht und damit auch die Aktivitäten der stärksten Oppositionspartei. Auch soll zu den durch Pegasus Überwachten ein bekannter Rechtsanwalt und Rechtsbevollmächtigter von Donald Tusk gehört haben. Außerdem tauchten Informationen darüber auf, dass der Vater des $P O$-Wahlkampfleiters Krzysztof Brejza sowie seine damalige Assistentin, heute Abgeordnete, wohl gehackt und abgehört wurden (bei der Enthüllung halfen Journalisten der deutschen Wochenzeitung »Die Zeit«). Immer häufiger werden in der öffentlichen Debatte in Polen Warnungen ausgesprochen, dass es bei den kommenden Parlamentswahlen zu vielfältigen Manipulationen und Missbrauch kommen kann, die zur Folge haben können, dass die Wahlen nicht gleich, möglicherweise nicht einmal frei sein werden. Es werden Befürchtungen lauter, dass das Regierungslager versuchen kann, die Wahlordnung zu seinen Gunsten zu verändern. Sollte die Opposition jedoch trotz dieser Hindernisse und Erschwernisse die Mehrheit im Parlament erlangen und die PiS aus der Regierung drängen, würde vermutlich keine Schwierigkeit mit der Bildung der Regierungskoalition bestehen. Die Oppositionsparteien eint das gemeinsame Bestreben, die Rechtsstaatlichkeit in Polen wiederherzustellen, den autoritären Praktiken ein Ende zu setzen und sie auszuschalten sowie die demokratischen und zivilgesellschaftlichen Institutionen zu erneuern. Das wären die Hauptaufgaben einer solchen künftigen Regierung.

Ein Hindernis wären hier allerdings das Vetorecht von Präsident Duda, dessen Amtszeit bis 2025 dauert, sowie zahlreiche Institutionen, die mit von der PiS nominiertem Personal ausgestattet sind, einschließlich dem
Verfassungstribunal, dessen Präsidentin Handlangerin der PiS und ihres Chefs Jarosław Kaczyński ist. Vorstellbar ist also die Situation, dass von der neuen parlamentarischen Mehrheit verabschiedete Gesetze vom Präsidenten mit Veto belegt oder vom Verfassungstribunal in Frage gestellt werden (der Präsident hat das Recht, sie dorthin zu verweisen). Vor diesem Hintergrund entstand, insbesondere vonseiten der $P O$, die politische Initiative "276«. 276 ist die erforderliche Anzahl der Mandate (zwei Drittel des Sejm), die es bedarf, um ein Veto des Präsidenten abzulehnen. Ein so hohes Ergebnis für die Opposition erscheint allerdings zurzeit unrealistisch (um die Regierung zu stellen, bedarf es 231 Abgeordnetensitze im Sejm).

Aufseiten der Opposition sind, wenngleich nicht allzu laute, Stimmen zu vernehmen, die dazu aufrufen, im aktuellen Sejm für eine Mehrheit zu sorgen, dass ein neues Kabinett mit Rückhalt aller parlamentarischen Fraktionen außerhalb des jetzigen Regierungslagers berufen werden kann. Möglich wäre das nur mit einem konstruktiven Misstrauensvotum - in Polen kann eine amtierende Regierung nur mit Hilfe der Einberufung einer neuen abgesetzt werden, was bedeutet, dass es für die neue Regierung eine parlamentarische Mehrheit geben muss. Hypothetisch ließe sich das machen, denn das aktuelle Regierungslager verfügt nur über 228 Stimmen, was ihm nicht die Mehrheit gibt, weshalb vor jeder Abstimmung die Unterstützung einiger unabhängiger Abgeordneter oder am Rande stehender, kleiner Abgeordnetenkreise gesucht wird. Bisher ist das den führenden Akteuren der Regierungsmannschaft gewöhnlich gelungen - auch oder insbesondere unter Zuhilfenahme von Methoden politischer Korruption, beispielsweise Versprechen vielfältiger Vorteile, Posten usw., die sich aus der Unterstützung für Machthaber ergeben, die über weitreichenden Einfluss verfügen. Jedoch traten für das Regierungslager auch mehrmals Niederlagen bei Abstimmungen im Sejm ein, wenngleich in der Regel bei weniger wesentlichen Fragen.

Als sehr wichtig, vielleicht sogar entscheidend für die Zukunft der aktuellen Regierungskoalition kann sich die für März angekündigte Abstimmung über die Einsetzung eines parlamentarischen Untersuchungsausschusses zur Aufklärung der mutmaßlichen Überwachung von Politikern der Opposition und Personen in ihrem Umfeld mit Hilfe der Kontrollsoftware Pegasus erweisen. Nicht ausgeschlossen ist die Unterstützung des Antrags von einzelnen Abgeordneten, die bislang das Regierungslager unterstützt haben, was für dieses eine empfindliche Niederlage bedeuten würde. Hinzu kommt, dass die Arbeit des Untersuchungsausschusses Einzelheiten ans Licht bringen könnte, die die Akteure des Regierungslagers kompromittieren könnten. Einige solcher Details wurden von einem bereits beru- 
fenen Senatsausschuss festgestellt, allerdings hat dieser weniger Kompetenzen und Befragungsmöglichkeiten.

Versuchen, im aktuellen Sejm eine Anti-Regierungsmehrheit zusammenzubringen, steht Donald Tusk ablehnend gegenüber, zumal er bedeutsame Erfahrungen gemacht hat. 2007, als die damals regierende PiS ihren Koalitionspartner und die parlamentarische Mehrheit verlor, wurde Tusk zugeredet, eine Regierung aus der ganzen damaligen Opposition zu bilden. Er ließ sich darauf nicht ein, es kam zu Neuwahlen, und diese gewann die $P O$, die anschließend zwei Wahlperioden zusammen mit der PSL regierte (Tusk bekleidete das Amt des Ministerpräsidenten sieben Jahre). Eine Wiederholung heute ist nicht ausgeschlossen.

Der wichtigste Grund für Spannungen und Risse im Regierungslager ist zurzeit das Verhältnis zur Europäischen Union. Ein Teil des insgesamt euroskeptischen rechtspopulistischen Lagers zeigt sich offen, sogar ostentativ antieuropäisch. In einer der am stärksten proeuropäisch eingestellten Gesellschaften Europas kann das zu einem Auslöser für politische Turbulenzen werden.

\section{Die Dilemmata der Opposition}

Die politische Opposition in Polen steht demzufolge vor einigen Dilemmata: auf die fortschreitende Zersetzung und einen möglichen Bruch des rechtspopulistischen Regierungslagers warten oder die Regierungsverantwortung noch vor Ablauf der regulären Wahlperiode zu übernehmen versuchen? Bis zu welchem Ausmaß innerhalb der Opposition um Wählerstimmen kämpfen und in welchem Umfang zusammenarbeiten? Sich auf gemeinsamen Wahllisten vereinigen oder zwei oder mehr eigenständige oppositionelle Blocks bilden? Mögliche Absprachen mit den oppositionellen Partnern und potentiellen Koalitionären schon jetzt ausloten oder auf Veränderungen im Kräfteverhältnis innerhalb der Opposition - möglicherweise erst infolge des Wahlergebnisses - warten? Versuchen, die bisherigen Wähler der Rechtspopulisten zu erreichen, was bedeuten würde, ihnen entgegengehen zu müssen, oder auf die Mobilisierung der eigenen Wähler setzen, also einen Schritt in die entgegengesetzte Richtung tun?

Leider schreitet die Zerstörung des demokratischen Rechtsstaates durch das aktuelle Regierungslager fort, so dass die Aufgaben, die vor einer möglicherweise siegreichen Opposition stehen, zunehmen, wenn sie das System der liberalen Demokratie in Polen wiederherstellen will.

Viel wird von den Signalen abhängen, welche die oppositionell eingestellte öffentliche Meinung den Anführern der Opposition senden wird. Sie zeigt eine zunehmende Ungeduld und fordert immer lauter die Vereinigung aller oppositionellen Kräfte. Stimmen, die zum gemeinsamen Handeln der politischen Opposition aufrufen, kommen auch von zivilgesellschaftlichen Organisationen, die gegen die autoritäre Politik der regierenden Rechtspopulisten eintreten. Sie organisieren u. a. Demonstrationen als Protest gegen die Aktivitäten des Regierungslagers und stellen damit einen bedeutsamen gesellschaftlichen Rückhalt für die politische Opposition dar.

Übersetzung aus dem Polnischen: Silke Plate 


\section{Parteipräferenzen und Politikerratings}

\section{Grafik 1: Parteipräferenzen}

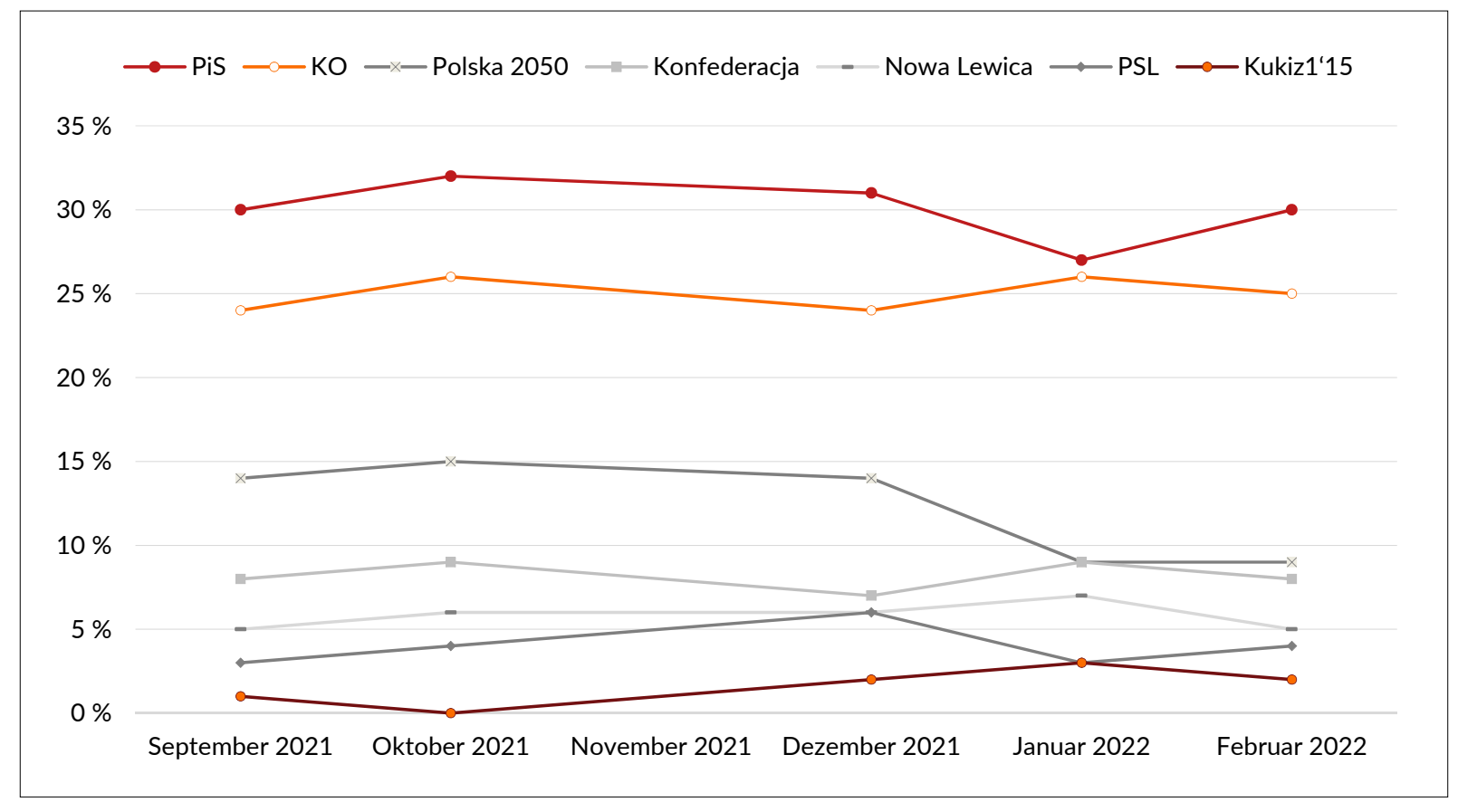

\begin{tabular}{|l|c|c|c|c|c|}
\hline & September 2021 & Oktober 2021 & Dezember 2021 & Januar 2022 & Februar 2022 \\
\hline PiS & $30 \%$ & $32 \%$ & $31 \%$ & $27 \%$ & $30 \%$ \\
\hline KO & $24 \%$ & $26 \%$ & $24 \%$ & $26 \%$ & $25 \%$ \\
\hline Kolska 2050 & $14 \%$ & $15 \%$ & $14 \%$ & $9 \%$ & $9 \%$ \\
\hline Nowfederacja & $8 \%$ & $9 \%$ & $7 \%$ & $9 \%$ & $8 \%$ \\
PSL & $5 \%$ & $6 \%$ & $6 \%$ & $7 \%$ & $4 \%$ \\
\hline Kukiz'15 & $3 \%$ & $4 \%$ & $6 \%$ & $3 \%$ & $2 \%$ \\
\hline
\end{tabular}

PiS/Prawo i Sprawiedliwość - Recht und Gerechtigkeit; KO/Koalicja Obywatelska - Bürgerkoalition; Polska 2050 - Polen 2050; Nowa Lewica - Neue Linke; PSL/Polskie Stronnictwo Ludowe - Polnische Bauernpartei

Quellen: Repräsentative Umfrage von Kantar Public, 09.-11.09.2021, in: Rzeczpospolita, 12.09.2021. https://www.rp.pl/polityka/art18910611-sondazkantar-pis-30-proc-koalicja-obywatelska-24-proc; repräsentative Umfrage von Kantar Public, in: Rzeczpospolita, 13.10.2021. https://www.rp.pl/ polityka/art19011861-sondaz-pis-6-pkt-proc-przed-ko-kukiz-15-z-poparciem-0-proc; repräsentative Umfrage von Kantar Public, 10.-12.12.2021, in: Rzeczpospolita, 13.12.2021. https://www.rp.pl/polityka/art19192521-sondaz-kantaru-szesc-partii-w-sejmie-psl-zrownalo-sie-z-lewica; repräsentative Umfrage von Kantar Public, 21.-26.01.2022, in: Rzeczpospolita, 31.01.2022. https://www.rp.pl/polityka/art34552231-sondaz-opozycjatuz-za-prawem-i-sprawiedliwoscia; repräsentative Umfrage von Kantar Public, 11.-16.02.2022, in: : Rzeczpospolita, 23.02.2022. https://www.rp.pl/ polityka/art35741271-sondaz-partyjny-niewielkie-wzrosty-niewielkie-spadki-prowadzi-pis (alle abgerufen am 25.02.2022). 


\section{Grafik 2: Politikerratings}

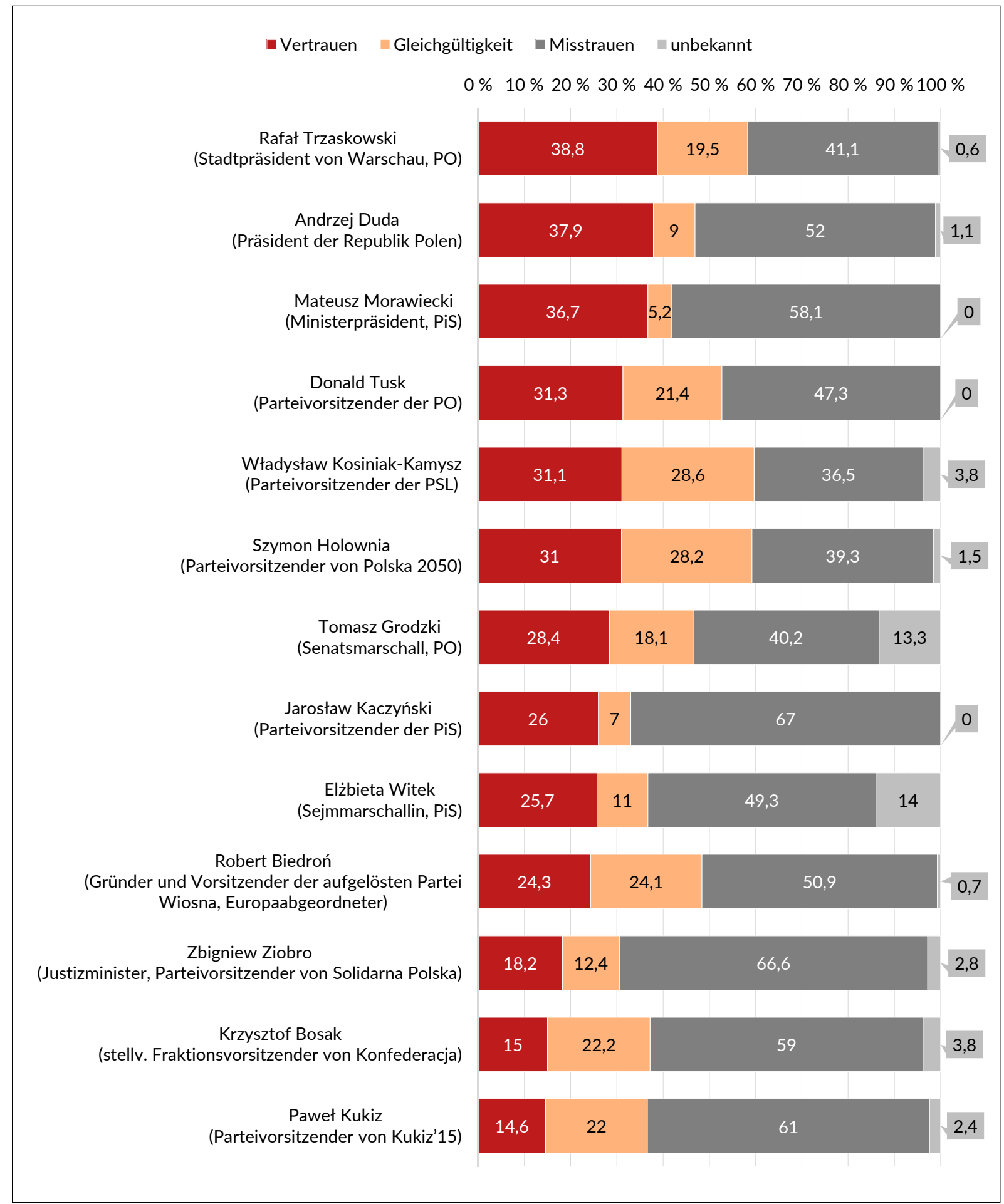

PO/Platforma Obywatelska - Bürgerplattform; PiS/Prawo i Sprawiedliwość - Recht und Gerechtigkeit; PSL/Polskie Stronnictwo Ludowe - Polnische Bauernpartei; Polska 2050 - Polen 2050; Wiosna - Frühling; Solidarna Polska - Solidarisches Polen; Konfederacja - Konföderation Umfrage von IBRiS, 11.-12.02.2022.

Quelle: Onet, 15.02.2022. https://www.onet.pl/informacje/onetwiadomosci/ranking-onetu-zaufania-do-politykow-trzaskowski-duda-i-morawieckina-czele/06tmdk0,79cfc278 (abgerufen am 25.02.2022). 


\section{5. - 28. Februar 2022}

\begin{tabular}{|c|c|}
\hline 15.02 .2022 & $\begin{array}{l}\text { In seiner Funktion als diesjähriger Vorsitzender der Organisation für Sicherheit und Zusammenarbeit in Europa } \\
\text { (OSZE) trifft sich Außenminister Zbigniew Rau in Moskau mit seinem russischen Amtskollegen Sergej Lawrow. } \\
\text { Rau wirbt für die Initiative Polens im Rahmen des polnischen OSZE-Vorsitzes, den europäischen Sicherheits- } \\
\text { dialog wiederzubeleben, sowie für die aktive Beteiligung Russlands an diesem Dialog. Thematisiert wird in die- } \\
\text { sem Zusammenhang auch die Sicherheitslage an der russisch-ukrainischen Grenze, wo Russland ein Truppen- } \\
\text { aufgebot von ca. } 120.000 \text { Soldaten zusammengezogen hat. Zum Abschluss seines Besuches in Moskau spricht } \\
\text { Rau mit Vertretern der russischen Zivilgesellschaft und Menschenrechtsexperten. }\end{array}$ \\
\hline 16.02 .2022 & $\begin{array}{l}\text { Der Europäische Gerichtshof (EuGH) weist die Klagen von Polen und Ungarn gegen den EU-Rechtsstaatsme- } \\
\text { chanismus ab. Der Mechanismus, der die Auszahlung von EU-Finanzmitteln an die EU-Mitgliedsländer von } \\
\text { deren Einhaltung rechtsstaatlicher Prinzipien abhängig macht, sei EU-rechtskonform und überschreite nicht die } \\
\text { Kompetenzen der Europäischen Union, so das Gericht in seiner Begründung. In einer ersten Reaktion bezeich- } \\
\text { net der Justizminister Polens, Zbigniew Ziobro (Solidarisches Polen/Solidarna Polska), den EuGH als politi- } \\
\text { sches Organ, das von der Europäischen Volkspartei (EVP) beeinflusst werde, die eine Verlängerung der deut- } \\
\text { schen Politik sei. Die Zustimmung zur Einführung des Rechtsstaatsmechanismus im Jahr } 2020 \text { sei ein gravie- } \\
\text { render politischer und historischer Fehler von Ministerpräsident Mateusz Morawiecki (Recht und Gerechtig- } \\
\text { keit/Prawo i Sprawiedliwość - PiS) gewesen, vor dem Solidarisches Polen damals gewarnt habe. Morawiecki } \\
\text { kontert, ein fundamentaler Fehler wäre es, wenn das Urteil zu Spannungen in der Regierungskoalition der Ver- } \\
\text { einigten Rechten (Zjednoczona Prawica) führen würde. }\end{array}$ \\
\hline 17.02 & $\begin{array}{l}\text { In einer gemeinsamen Erklärung aus Anlass des massiven russischen Truppenaufmarsches an der russisch- } \\
\text { ukrainischen Grenze geben Großbritannien, Polen und die Ukraine ihre Absicht bekannt, eine Vereinbarung } \\
\text { über eine trilaterale strategische Zusammenarbeit (u. a. in den Bereichen Cybersicherheit, Abwehr von Desin- } \\
\text { formationsangriffen und Energiesicherheit) auszuarbeiten. }\end{array}$ \\
\hline 18.02 & $\begin{array}{l}\text { In Międzyrzecz (Woiwodschaft Lebuser Land/województwo lubuskie) beginnt das multinationale Militärmanö- } \\
\text { ver Saber Strike-22, das u. a. in Polen und den baltischen Staaten stattfindet und bis zum 6. März dauern soll. } \\
\text { Aus Polen nehmen ca. } 1.300 \text { Soldaten teil. Gegenstand der Übung ist die Verlegung von Truppen und Gerät } \\
\text { aus den USA nach Europa und die Abwehr militärischer Gefahren an der NATO-Ostgrenze. }\end{array}$ \\
\hline 18.02 .2022 & $\begin{array}{l}\text { Am letzten Tag seines zweitägigen Besuchs in der Ukraine spricht sich Senatsmarschall Tomasz Grodzki (Bür- } \\
\text { gerplattform/Platforma Obywatelska - PO) in Kiew für eine schnelle Assoziierung der Ukraine an die NATO } \\
\text { und die Europäische Union aus, sobald die Hochphase des russisch-ukrainischen Konflikts überwunden ist. } \\
\text { Die EU solle ein Hilfspaket für die ukrainische Wirtschaft in Erwägung ziehen, denn viele ausländische Inves- } \\
\text { toren hätten sich infolge der militärischen Krise aus der Ukraine zurückgezogen. Grodzki wird von einer Dele- } \\
\text { gation des Senats der Republik Polen begleitet. Neben einer Rede Grodzkis vor dem Obersten Nationalrat } \\
\text { der Ukraine fanden Gespräche der Senatoren u. a. mit dem Präsidenten des ukrainischen Parlaments, Ruslan } \\
\text { Stefantschuk, statt. }\end{array}$ \\
\hline 18.02 .2022 & $\begin{array}{l}\text { Szymon Szynkowski vel Sęk, Staatssekretär im Außenministerium, sagt bei seinem Besuch in Berlin in Bezug } \\
\text { auf die russisch-ukrainische Krise, Polen erwarte - mit Blick auf Sanktionen des Westens gegenüber Russland } \\
\text { sowie die Wünsche der Ukraine nach Unterstützung - konkrete Handlungen von Deutschland und nicht nur } \\
\text { Erklärungen. }\end{array}$ \\
\hline 19.02 .2022 & $\begin{array}{l}\text { Auf der Münchener Sicherheitskonferenz erklärt Ministerpräsident Mateusz Morawiecki die Bereitschaft Polens, } \\
\text { der Ukraine weitere Defensivwaffen für den möglichen Ernstfall im russisch-ukrainischen Konflikt zu liefern. } \\
\text { Man dürfe die Einstellung Russlands nicht akzeptieren, dass manche europäischen Staaten ein größeres Recht } \\
\text { auf Frieden und Sicherheit hätten als andere. Unabhängig von ihrer Stärke oder Schwäche hätten alle souverä- } \\
\text { nen Staaten dasselbe Recht, in Frieden zu leben. }\end{array}$ \\
\hline 19.02 .2022 & $\begin{array}{l}\text { Aufgrund der angespannten Sicherheitslage in der Ukraine infolge des russisch-ukrainischen Konflikts rät das } \\
\text { Außenministerium von Reisen in die Südostukraine ab und fordert die polnischen Staatsbürger, die sich dort } \\
\text { aufhalten, auf, ihre Rückkehr in Betracht zu ziehen. Außerdem wird von nicht notwendigen Reisen in die übri- } \\
\text { gen Gebiete der Ukraine abgeraten. }\end{array}$ \\
\hline 20.02 .2022 & $\begin{array}{l}\text { Die stellvertretende Bürgerrechtsbeauftragte, Hanna Machińska, sagt in einem Interview mit TVN24, Polen } \\
\text { müsse sich organisatorisch und infrastrukturell darauf vorbereiten, Flüchtlinge aus der Ukraine bei einem } \\
\text { potentiellen Einmarsch Russlands in die Ukraine aufzunehmen. Dabei gehe es nicht nur um die kurzfristige } \\
\text { Versorgung der Menschen, sondern auch darum, ihnen langfristig eine normale Existenz in Polen zu gewähr- } \\
\text { leisten. Die schlechten Erfahrungen mit der Migrationskrise an der polnisch-belarussischen Grenze, die seit } \\
\text { einem Dreivierteljahr besteht, dürften sich nicht wiederholen. }\end{array}$ \\
\hline
\end{tabular}




\begin{tabular}{|c|c|}
\hline 21.02 .2022 & $\begin{array}{l}\text { Das Außenministerium verurteilt entschieden die Anerkennung der selbsternannten »Volksrepubliken Donezk» } \\
\text { und »Lugansk« in der Ostukraine durch Russland. Die »Volksrepubliken« werden von Separatisten kontrolliert. } \\
\text { Die Anerkennung bedeute einen Bruch mit den Minsker Abkommen, das Russland mitunterzeichnet hat, und } \\
\text { sei eine Verletzung des Völkerrechtes. Polen sagt der Ukraine seine Solidarität zu und ruft Russland zur Dees- } \\
\text { kalation und zum Truppenabzug von der russisch-ukrainischen Grenze auf. }\end{array}$ \\
\hline 22.02 .2022 & $\begin{array}{l}\text { Nach der Anerkennung der selbsternannten »Volksrepubliken Donezk« und »Lugansk« in der Ostukraine durch } \\
\text { Russland am Vortag ruft Polen als Vorsitzender der Organisation für Sicherheit und Zusammenarbeit in Europa } \\
\text { (OSZE) eine Sondersitzung des Ständigen Rates ein. Vizeaußenminister Marcin Przydacz verurteilt die Ent- } \\
\text { scheidung Russlands und unterstreicht, dass es keine Alternative zu einer diplomatischen Lösung der Span- } \\
\text { nungen auf dem Gebiet der OSZE gebe. }\end{array}$ \\
\hline 22.02 .2022 & $\begin{array}{l}\text { Laut einem Entwurf des Innenministeriums soll das Aufenthaltsverbot im Grenzstreifen zu Belarus über den } \\
\text { 1. März hinaus bis zum 30. Juni } 2022 \text { verlängert werden. Das Verbot besteht seit dem 1. Dezember } 2021 \text { und } \\
\text { wurde infolge der Migrationskrise an der belarussischen Grenze verhängt. }\end{array}$ \\
\hline 23.02 .2022 & $\begin{array}{l}\text { Die Delegation aus elf Abgeordneten des Europäischen Parlaments, die zur Beurteilung der Lage der Rechts- } \\
\text { staatlichkeit in Polen entsandt wurde, beendet ihren dreitägigen Aufenthalt in Polen. Auf einer Pressekonfe- } \\
\text { renz unterstreicht Juan Fernando Lopez Aguilar, Ausschuss des EU-Parlaments für bürgerliche Freiheiten, Jus- } \\
\text { tiz und Inneres, dass die Europäische Union nicht nur ein gemeinsamer Markt, sondern vor allem eine Werte- } \\
\text { gemeinschaft sei, deren Einhaltung durch den Europäischen Gerichtshof (EuGH) beurteilt wird. Seit Inkraft- } \\
\text { treten des Lissabonner Vertrags sei die Rechtsstaatlichkeit nicht mehr nur eine nationale, sondern eine euro- } \\
\text { päische Frage. In Polen sei die Erosion der Unabhängigkeit der Justiz festzustellen. Othmar Karas, Ausschuss } \\
\text { für konstitutionelle Fragen, betont, vor dem Hintergrund der aktuellen russisch-ukrainischen Krise sei es umso } \\
\text { wichtiger, die grundlegenden europäischen Werte wie Demokratie, Rechtsstaatlichkeit, Menschenrechte und } \\
\text { Grundrechte einzuhalten. Er ruft die Europäische Kommission auf, keine Finanzhilfen für den Landesaufbau- } \\
\text { plans Polens (zur Bekämpfung der Folgen der Corona-Pandemie) auszuzahlen, bevor das Urteil des EuGH, dass } \\
\text { die Disziplinarkammer beim Obersten Gericht (Sąd Najwyższy - SN) aufgelöst werden muss, nicht umgesetzt } \\
\text { worden sei. Er sagt, es sei beunruhigend, dass Vertreter des Regierungslagers in Polen keine Bereitschaft gezeigt } \\
\text { hätten, sich mit den Delegierten des EU-Parlaments zu treffen. Ein solches Verhalten hätten auch regierungs- } \\
\text { kritische zivilgesellschaftliche Organisationen in Polen beklagt. }\end{array}$ \\
\hline 24.02 & $\begin{array}{l}\text { Das Außenministerium verurteilt entschieden den militärischen Angriff Russlands auf verschiedene Ziele in } \\
\text { der Ukraine, der heute begann. Dies sei ein präzedenzloser Vorfall und die bewusst getroffene Entscheidung } \\
\text { Russlands, die Grundlagen der gegenwärtigen Sicherheitsarchitektur zu zerstören und Staatsgrenzen gewalt- } \\
\text { sam zu verschieben. Im 21. Jahrhundert sei dies nicht akzeptabel. Polen unterstütze gemeinsam mit den west- } \\
\text { lichen Partnern die Ukraine. }\end{array}$ \\
\hline 25.02 .2022 & $\begin{array}{l}\text { Auf Initiative von Präsident Andrzej Duda findet in Warschau ein Sondergipfel der »Bukarester Neun« (Bulga- } \\
\text { rien, Estland, Lettland, Litauen, Polen, Rumänien, Slowakei, Tschechien und Ungarn) statt, an dem außer den } \\
\text { Staatschefs auch die Präsidentin der Europäischen Kommission, Ursula von der Leyen, teilnimmt. Während } \\
\text { des Treffens wird eine online-Verbindung zum NATO-Sondergipfel hergestellt. Angesichts des russischen Mili- } \\
\text { tärangriffs auf die Ukraine fordert Duda mehr konkrete militärische, wirtschaftliche, finanzielle und humani- } \\
\text { täre Hilfe für die Ukraine. Die beiden Gipfel wertet Duda als wichtige Beiträge zur Diskussion über tiefgrei- } \\
\text { fende Sanktionen gegenüber Russland. }\end{array}$ \\
\hline 25.02 .2022 & $\begin{array}{l}\text { Regierungssprecher Piotr Müller gibt bekannt, dass Polen als Antwort auf den am Vortag begonnenen Angriffs- } \\
\text { krieg Russlands auf die Ukraine seinen Luftraum für russische Flugzeuge schließt. }\end{array}$ \\
\hline 25.02 .2022 & $\begin{array}{l}\text { Regierungssprecher Piotr Müller teilt mit, dass Polen und die Ukraine einen gemeinsamen Antrag auf Ausset- } \\
\text { zung der Mitgliedschaft Russlands im Europarat gestellt haben. Hintergrund ist der Einmarsch Russlands in } \\
\text { die Ukraine am Vortag. }\end{array}$ \\
\hline 25.02 .2022 & $\begin{array}{l}\text { Der stellvertretende Innenminister Paweł Szefernaker informiert, dass am Vortag } 31.000 \text { Personen infolge des } \\
\text { russischen Militärangriffs auf die Ukraine die Grenze nach Polen überschritten haben. Am heutigen Tag seien } \\
\text { es bis } 15 \text { Uhr } 25.000 \text { Menschen gewesen. An den zentralen Busbahnhöfen in den Hauptstädten der Woiwod- } \\
\text { schaften und am Flughafen in Warschau würden Informationsstellen für Flüchtlinge eingerichtet. }\end{array}$ \\
\hline 25.02 .2022 & $\begin{array}{l}\text { Als Reaktion auf den russischen Angriffskrieg auf die Ukraine ruft die Wirtschafts- und Handelskammer des } \\
\text { Lebensmittelmarktes (Izba Gospodarczo Handlowa Rynku Spożywczego) dazu auf, keine russischen und bela- } \\
\text { russischen Produkte zu kaufen. }\end{array}$ \\
\hline
\end{tabular}




\begin{tabular}{|c|c|}
\hline 26.02 .2022 & $\begin{array}{l}\text { Vor dem Treffen mit Bundeskanzler Olaf Scholz in Berlin kündigt Ministerpräsident Mateusz Morawiecki in } \\
\text { einem Briefing an, er wolle das Gewissen Deutschlands erschüttern, mit dem Ziel, dass sich Deutschland für } \\
\text { "vernichtende« Sanktionen gegenüber Russland entscheide. Damit die EU von russischen Energielieferungen } \\
\text { unabhängig werde, müsse nicht nur auf die Gaspipeline Nord Stream 2, sondern auch Nord Stream } 1 \text { verzich- } \\
\text { tet werden. Weiter müsse die russische Finanzwirtschaft aus dem SWIFT-System ausgeschlossen und der rus- } \\
\text { sische Präsident Wladimir Putin persönlich mit Sanktionen belegt werden. Die Lieferung Deutschlands von } \\
5.000 \text { Militärhelmen an die Ukraine bezeichnet Morawiecki als "Witz« und fordert reelle Hilfe in Form von } \\
\text { Waffenlieferungen Deutschlands. }\end{array}$ \\
\hline 26.02 & $\begin{array}{l}\text { Bundeskanzler Olaf Scholz empfängt Ministerpräsident Mateusz Morawiecki und den litauischen Staatspräsi- } \\
\text { denten Gitanas Nausèda in Berlin zu einem Gespräch über die Invasion Russlands in die Ukraine, die vor zwei } \\
\text { Tagen begann. Der Angriffskrieg der Russischen Föderation gegen die Ukraine sei durch nichts zu rechtferti- } \\
\text { gen. Gewürdigt wurden die Einheit der EU und die schnell beschlossenen, umfangreichen Sanktionen der EU } \\
\text { gegenüber Russland. Weitere zielgerichtete Maßnahmen sollen vereinbart werden. Thematisiert wurde auch } \\
\text { die Notwendigkeit, die NATO-Ostflanke zu verstärken, heißt es in der Pressemitteilung des Bundeskanzler- } \\
\text { amtes nach dem Treffen. }\end{array}$ \\
\hline 27.02 & $\begin{array}{l}\text { Der stellvertretende Innenminister Błażej Poboży teilt mit, dass ein von Polen eingerichteter medizinischer } \\
\text { Eisenbahntransport auf seiner Probefahrt ca. } 600 \text { Flüchtlinge (Frauen und Kinder) aus der Ukraine nach Polen } \\
\text { gebracht hat. Der für medizinische Zwecke eingerichtete Zug soll infolge des Kriegsgeschehens zwischen Russ- } \\
\text { land und der Ukraine Verletzte aus der Ukraine nach Polen bringen, wo sie ärztlich versorgt werden. }\end{array}$ \\
\hline 27.02 .2022 & $\begin{array}{l}\text { Tomasz Praga, Oberbefehlshaber des Grenzschutzes, informiert, dass seit dem Einmarsch russischer Truppen } \\
\text { in die Ukraine am 24. Februar knapp } 200.000 \text { Flüchtlinge aus der Ukraine Hilfe in Polen erhalten hätten. }\end{array}$ \\
\hline 28.02 .2022 & $\begin{array}{l}\text { In einem Interview in der spanischen Zeitung Corriere della Sera fordert Ministerpräsident Mateusz Morawiecki } \\
\text { angesichts des russischen Militärangriffs auf die Ukraine, dass alle östlichen NATO-Mitgliedsstaaten mit mehr } \\
\text { Militär sowie Raketenabwehrsystemen ausgerüstet werden müssen. Der russische Präsident Wladimir Putin } \\
\text { werde seine aggressive Politik des Wiederaufbaus des russischen Imperiums möglicherweise beschleunigen. Nach } \\
\text { Georgien und aktuell der Ukraine könnten die baltischen Staaten, Polen, Finnland und andere ostmitteleuro- } \\
\text { päische Staaten sein Ziel werden. Morawiecki spricht sich dafür aus, die Verteidigungsausgaben der Europäi- } \\
\text { schen Union von zurzeit ca. } 300 \text { Mrd. Euro auf 500-600 Mrd. Euro zu steigern. }\end{array}$ \\
\hline
\end{tabular}

Sie können die gesamte Chronik seit 2007 auch auf http://www.laender-analysen.de/polen/ unter dem Link "Chronik« lesen. 


\section{ÜBER DIE POLEN-ANALYSEN}

Die Polen-Analysen erscheinen zweimal monatlich als E-Mail-Dienst. Sie werden gemeinsam vom Deutschen PolenInstitut Darmstadt, der Forschungsstelle Osteuropa an der Universität Bremen und weiteren Partnern eines Konsortiums (siehe Titelseite) herausgegeben.

Ein Archiv der Polen-Analysen finden Sie im Internet unter www.laender-analysen.de/polen Kostenloses Abonnement unter http://www.deutsches-polen-institut.de/Newsletter/subscribe.php

Diese Analysen finden Sie online als Lizenzausgabe auf bpb.de

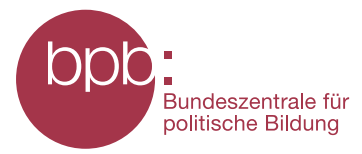

Deutsches Polen-Institut Darmstadt (www.deutsches-polen-institut.de)

Das seit 1980 tätige Deutsche Polen-Institut Darmstadt (DPI) ist ein Forschungs-, Informations- und Veranstaltungszentrum für polnische Kultur, Geschichte, Politik, Gesellschaft und die deutsch-polnischen Beziehungen, die sich im Kontext der europäischen Integration entwickeln. Institutionelle Träger des DPI sind das Land Hessen, die Kultusminister der Länder, das Auswärtige Amt und die Wissenschaftsstadt Darmstadt. Einen wesentlichen Beitrag zur Verwirklichung der Institutsziele leisten private Stiftungen. Ziel der Vermittlertätigkeit des DPI ist es, "die zu interessieren, auf die es politisch, wirtschaftlich, gesellschaftlich und kulturell im deutsch-polnischen Verhältnis ankommt (Leitlinien 1997). Es geht um die Entscheider und Multiplikatoren in Politik, Kultur, Bildung, Verwaltung, Medien und Wirtschaft. Das DPI versteht sich in Kooperation mit den Orten wissenschaftlicher Polen-Kompetenz an deutschen Hochschulen und Forschungsinstituten als verbindendes und vernetzendes Zentrum. Mit der 70.000 Bände zählenden multidisziplinären Fachbibliothek für Polen, die eine einzigartige Sammlung polnischer Belletristik in der Originalsprache und in deutscher Übersetzung umfasst, ist das DPI ein geschätzter Ort der Recherche und des wissenschaftlichen Arbeitens.

Forschungsstelle Osteuropa an der Universität Bremen (www.forschungsstelle.uni-bremen.de)

1982 gegründet, widmet sich die Forschungsstelle Osteuropa an der Universität Bremen der interdisziplinären Analyse der Länder Ost- und Ostmitteleuropas in Zeitgeschichte und Gegenwart. Der Forschungsschwerpunkt liegt dabei auf der Rolle von »Dissens und Konsens«, von Opposition und Zivilgesellschaft in ihrem historischen, politischen, gesellschaftlichen und kulturellen Kontext. Die Forschungsstelle besitzt in ihrem Archiv eine einzigartige Sammlung alternativer Kulturgüter und unabhängiger Texte aus den ehemaligen sozialistischen Ländern. Darunter befindet sich auch eine umfangreiche Sammlung des "Zweiten Umlaufs«, die das Schrifttum und Dokumente unabhängiger Initiativen und gesellschaftlicher Gruppen in Polen aus der Zeit von 1976 bis zum Umbruch umfasst. Hinzu kommt eine umfangreiche Bibliothek mit wissenschaftlicher Literatur. Mit Archiv, Bibliothek und zwei wissenschaftlichen Abteilungen ist die Forschungsstelle auch eine Anlaufstelle sowohl für Gastwissenschaftler als auch für die interessierte Öffentlichkeit.

Eine der Hauptaufgaben der Forschungsstelle ist die Information der interessierten Öffentlichkeit. Dazu gehören unter anderem regelmäßige E-Mail-Informationsdienste für Politik, Wirtschaft, Zivilgesellschaft und Medien.

Herausgeber:

Deutsches Polen-Institut, Deutsche Gesellschaft für Osteuropakunde e.V., Forschungsstelle Osteuropa an der Universität Bremen, Leibniz-Institut für Agrarentwicklung in Transformationsökonomien, Leibniz-Institut für Ost- und Südosteuropaforschung, Zentrum für Osteuropa- und internationale Studien (Z0iS) gGmbH

Redaktion:

Dr. Andrzej Kaluza (verantwortlich) (Darmstadt) und Dr. Silke Plate (Bremen)

Satz: Matthias Neumann

Wissenschaftlicher Beirat:

Prof. Dr. Stefan Garsztecki, Technische Universität Chemnitz

Prof. Dr. Klaus Ziemer, Kardinal-Stefan-Wyszyński-Universität Warschau

Die Meinungen, die in den Polen-Analysen geäußert werden, geben ausschließlich die Auffassung der Autoren wieder.

Abdruck und sonstige publizistische Nutzung sind nach Rücksprache mit der Redaktion gestattet. Polen-Analysen-Layout: Cengiz Kibaroglu, Matthias Neumann

Alle Ausgaben der Polen-Analysen sind mit Themen- und Autorenindex archiviert unter www.laender-analysen.de

ISSN 1863-9712 @ 2022 by Deutsches Polen-Institut, Deutsche Gesellschaft für Osteuropakunde e.V., Forschungsstelle Osteuropa an der Universität Bremen, Leibniz-Institut für Agrarentwicklung in Transformationsökonomien, Leibniz-Institut für Ost- und Südosteuropaforschung, Zentrum für Osteuropa- und internationale Studien (Z0iS) gGmbH Kontakt: Dr. Andrzej Kaluza, Deutsches Polen-Institut, Residenzschloss, Marktplatz 15, 64283 Darmstadt,

Tel.: +49/6151/4202-20, Fax: +49/6151/4202-10, E-Mail: kaluza@dpi-da.de, Internet:www.laender-analysen.de/polen 


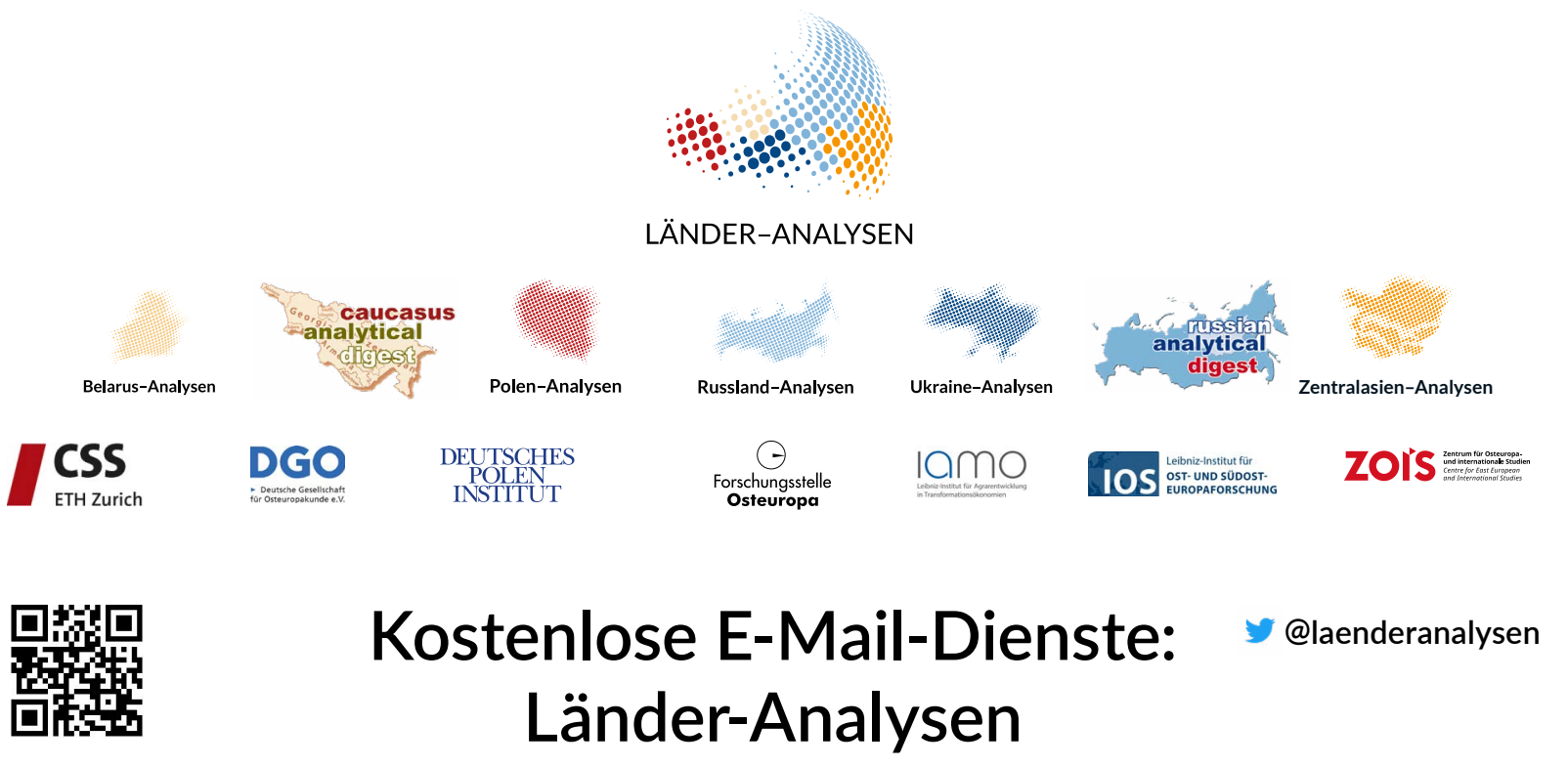

Die Länder-Analysen bieten regelmäßig im kostenlosen Abonnement kompetente Einschätzungen aktueller politischer, wirtschaftlicher, sozialer und kultureller Entwicklungen in Ostmitteleuropa und der GUS. Alle Länder-Analysen verstehen sich als Teil eines gemeinsamen Projektes, das der wissenschaftlich fundierten, allgemeinverständlich formulierten Analyse der Entwicklungen im östlichen Europa, der Offenheit für verschiedene inhaltliche Positionen und der kostenlosen und nicht-kommerziellen Information einer breit verstandenen interessierten Öffentlichkeit verpflichtet ist. Autor/innen sind internationale Fachwissenschaftler/innen und Expert/innen. Die Redaktionen der Länder-Analysen bestehen aus Wissenschaftler/innen mit langjähriger Forschungserfahrung.

Die deutschsprachigen Länder-Analysen werden gemeinsam von der Forschungsstelle Osteuropa an der Universität Bremen, dem Zentrum für Osteuropa- und internationale Studien, der Deutschen Gesellschaft für Osteuropakunde, dem Deutschen Polen-Institut, dem Leibniz-Institut für Agrarentwicklung in Transformationsökonomien und dem Leibniz-Institut für Ost- und Südosteuropaforschung herausgegeben. Die englischsprachigen Länder-Analysen erscheinen in Kooperation der Forschungsstelle Osteuropa mit dem Center for Security Studies (CSS) der ETH Zürich.

Die Länder-Analysen bieten regelmäßig Kurzanalysen zu aktuellen Themen, ergänzt um Grafiken und Tabellen sowie Dokumentationen. Zusätzlich gibt es eine Chronik aktueller Ereignisse.

\section{Belarus-Analysen}

Erscheinungsweise: zweimonatlich

Abonnement unter: http://www.laender-analysen.de/belarus/

\section{Caucasus Analytical Digest}

In englischer Sprache. Erscheinungsweise: zweimonatlich

Abonnement unter: http://www.css.ethz.ch/en/publications/cad.html

\section{Polen-Analysen}

Erscheinungsweise: zweimal monatlich

Abonnement unter: http://www.deutsches-polen-institut.de/newsletter/polen-analysen/

\section{Russland-Analysen}

Erscheinungsweise: zweimal monatlich

Abonnement unter: http://www.laender-analysen.de/russland/

\section{Russian Analytical Digest}

In englischer Sprache. Erscheinungsweise: zweimal monatlich Abonnement unter: http://www.css.ethz.ch/en/publications/rad.html

\section{Ukraine-Analysen}

Erscheinungsweise: zweimal monatlich

Abonnement unter: http://www.laender-analysen.de/ukraine/

\section{Zentralasien-Analysen}

Erscheinungsweise: zweimonatlich

Abonnement unter: http://www.laender-analysen.de/zentralasien/ 